\title{
Mejora docente aplicada al tema "Cultura, Clima y Comunicación en las Organizaciones" en la asignatura Psicología del Trabajo y de las Organizaciones
}

Francisco José SANCLEMENTE IBÁÑEZ

Universidad de Sevilla

Departamento de Psicología Social

fsanclemente@us.es

D.O.I.: http://dx.doi.org/10.12795/JDU.2018.i01.45

Pp.: 801-815

\section{Resumen}

Este ciclo de mejora docente es resultado del Curso General de Docencia Universitaria (CGDU) organizado por el Instituto de Ciencias de la Educación (ICE) de la Universidad de Sevilla. El ciclo de mejora se realizó con el tema "Cultura, clima y comunicación en las Organizaciones" dentro de la asignatura Psicología del Trabajo y de las Organizaciones en primer curso del Grado de Relaciones Laborales. La metodología didáctica se basó en el Modelo de Reelaboración de Ideas y en los Talleres Conceptuales, tratando a su vez de introducir mejoras en las habilidades docentes. Los resultados fueron satisfactorios, tanto respecto a la mejora del aprendizaje de los alumnos como en lo relativo a la propia mejora del docente. 
Palabras clave: Psicología del Trabajo y de las Organizaciones, Grado en Relaciones Laborales, Docencia universitaria, Experimentación docente universitaria, Mejora docente.

\section{Introducción y contextualización}

Este trabajo es el resultado de la aplicación de las diferentes tareas propuestas en las sesiones del Curso General de Docencia Universitaria (CGDU) organizado por el Instituto de Ciencias de la Educación (ICE) de la Universidad de Sevilla durante los meses de marzo, abril y mayo de 2018. Durante el curso se diseñaron y aplicaron dos ciclos de mejora docente; en este trabajo se detalla el segundo ciclo de mejora docente.

Este segundo ciclo tuvo una duración de 6 horas docentes en total, divididas en 3 sesiones de 2 horas cada una, y se realizó con el tema "Cultura, Clima y Comunicación en las Organizaciones" dentro de la asignatura Psicología del Trabajo y de las Organizaciones en primer curso del Grado de Relaciones Laborales en la Facultad de Ciencias del Trabajo de la Universidad de Sevilla, con un total de 54 alumnos matriculados. A su vez, este tema presenta tres bloques bien definidos, el primero se dedica a la cultura organizacional, el segundo al clima organizacional y el tercero y último a la comunicación organizacional. Se trata, pues, de contenidos clave para la formación de futuros profesionales dentro del campo de las Relaciones Laborales en las organizaciones. Los contenidos se apoyaron en el manual de Gil y Alcover (2003), Introducción a la Psicología de las Organizaciones. Hay que señalar que el aula donde se desarrolló el ciclo tiene la típica distribución de bancadas en hileras, que se adapta bien a las clases expositivas pero que ofrece ciertas barreras a los trabajos en grupo.

Jornadas de Formación e Innovación Docente del Profesorado | № 1 (2018) Esta obra se distribuye con la licencia Creative Commons 


\section{Modelo metodológico y principios didácticos que han guiado la experiencia}

En este ciclo de mejora docente, se utilizó, como referencia básica, el Modelo de Reelaboración de Ideas planteado en el capítulo 2 (De Alba y Porlán, 2017) del libro Enseñanza universitaria. Cómo mejorarla, coordinado por Rafael Porlán. Este modelo presenta 4 fases bien definidas: Pr, le, Ac y C. Pr: planteamiento de un problema de interés. le: toma de conciencia de las ideas del alumnado. Ac: puesta en práctica de actividades de contraste que pongan en cuestión las ideas de los alumnos. C: elaboración de conclusiones en relación con las ideas iniciales y finales y sobre lo aprendido. Por otra parte, se aplicó simultáneamente la estrategia metodológica de los Talleres Conceptuales de Finkel (2008) relativa a la creación de esquemas para el aprendizaje mediante secuencias de preguntas, en grupos de cuatro alumnos, dando las instrucciones por escrito y guiando a los alumnos en su trabajo en grupo centrando la atención así en el problema planteado.

Adicionalmente, se aplicaron los principios propuestos por Ken Bain (2007) para mejorar el éxito de la docencia: crear un entorno para el aprendizaje crítico natural; conseguir la atención de los alumnos y no perderla; comenzar con los estudiantes en lugar de con la disciplina; buscar compromisos; ayudar a los estudiantes a aprender fuera de clase; atraer a los estudiantes al razonamiento disciplinar; crear experiencias de aprendizaje diversas.

Y también se introdujeron mejoras en el oficio docente y las habilidades técnicas y físicas por parte del profesor para favorecer el aprendizaje de los alumnos, tratando de mejorar la oratoria, usando un lenguaje cálido y que implique a los alumnos, dando explicaciones comenzando por generalizaciones simples usando un lenguaje familiar y avanzando posteriormente hacia la complejidad, dejando que hablen los estudiantes y planteando preguntas críticas y originales.

Respecto a la metodología de evaluación, en este ciclo de mejora se evaluaron tanto los conocimientos adquiridos por los alumnos y alumnas en clase, durante y al final 
del ciclo, como el oficio docente y las habilidades del profesor al finalizar el ciclo.

\section{Diseño del Ciclo de Mejora Docente}

El diseño de este segundo ciclo de mejora docente se plasma a nivel general en la Tabla 1. Consta de cuestionarios inicial y final y tres bloques, cada uno dedicado a uno de los tres conceptos principales del tema (cultura, clima y comunicación).

Tabla 1. Diseño del segundo ciclo de mejora docente.

\begin{tabular}{|c|c|}
\hline \multicolumn{2}{|r|}{ DISEÑO DEL SEGUNDO CICLO DE MEJORA DOCENTE } \\
\hline 1 & $\begin{array}{l}\text { Cuestionario previo pre-test de evaluación de los esquemas } \\
\text { mentales }\end{array}$ \\
\hline 2 & Introducción del tema \\
\hline 3 & Bloque 1: Cultura \\
\hline 4 & $\begin{array}{l}\text { Introducción del bloque à Modelo de reelaboración de las ideas para } \\
\text { cada concepto importante del bloque à Puesta en común à Resumen } \\
\text { del bloque }\end{array}$ \\
\hline 5 & Autoevaluación de conocimientos del bloque 1 \\
\hline 6 & Bloque 2: Clima \\
\hline 7 & $\begin{array}{l}\text { Introducción del bloque à Modelo de reelaboración de las ideas para } \\
\text { cada concepto importante del bloque à Puesta en común à Resumen } \\
\text { del bloque }\end{array}$ \\
\hline 8 & Autoevaluación de conocimientos del bloque 2 \\
\hline 9 & Bloque 3: Comunicación \\
\hline 10 & $\begin{array}{l}\text { Introducción del bloque à Modelo de reelaboración de las ideas para } \\
\text { cada concepto importante del bloque à Puesta en común à Resumen } \\
\text { del bloque }\end{array}$ \\
\hline 11 & Autoevaluación de conocimientos del bloque 3 \\
\hline 12 & Resumen del tema \\
\hline 13 & $\begin{array}{l}\text { Cuestionario final pos-test de evaluación de los esquemas mentales: } \\
\text { Taller conceptual caso empresa Malrollo S.A. }\end{array}$ \\
\hline 14 & Cuestionario de evaluación de docencia y habilidades docentes \\
\hline
\end{tabular}

Jornadas de Formación e Innovación Docente del Profesorado | № 1 (2018) Esta obra se distribuye con la licencia Creative Commons Reconocimiento-NoComercial-SinObraDerivada Internacional (CC BY-NC-ND 4.0.) 


\section{Mapa de contenidos y actividades}

El mapa de contenidos y actividades de este segundo ciclo de mejora docente (Figura 1) se estructura en los tres bloques antes citados. Cada bloque corresponde al concepto principal del mismo: Cultura, Clima y Comunicación respectivamente. En el mapa se señala mediante un sistema de colores los cuatro tipos de actividades propuestas: inicio y finalización (verde), reflexión o trabajo (azul), expositivas (naranja), y evaluación (morado).

\section{Desarrollo previsto del ciclo de mejora docente}

Antes de comenzar con el primero de los tres bloques citados, se pasa un cuestionario previo (pre-test) para evaluar los esquemas mentales de los alumnos. A continuación, se realiza una presentación general del tema y posteriormente se desarrolla los diversos bloques, manteniendo cada bloque una estructura común, como puede verse en la Tabla 2.

Tabla 2. Estructura de cada bloque del segundo ciclo de mejora docente.

\begin{tabular}{|l|}
\hline \multicolumn{1}{|c|}{ Estructura del ciclo de mejora } \\
\hline Introducción al bloque \\
\hline
\end{tabular}

Esquema de resolución de problemas con cada uno de los contenidos del bloque

Contenidos del bloque

Puesta en común

Resumen del bloque

Autoevaluación

Jornadas de Formación e Innovación Docente del Profesorado | № 1 (2018) Esta obra se distribuye con la licencia Creative Commons 


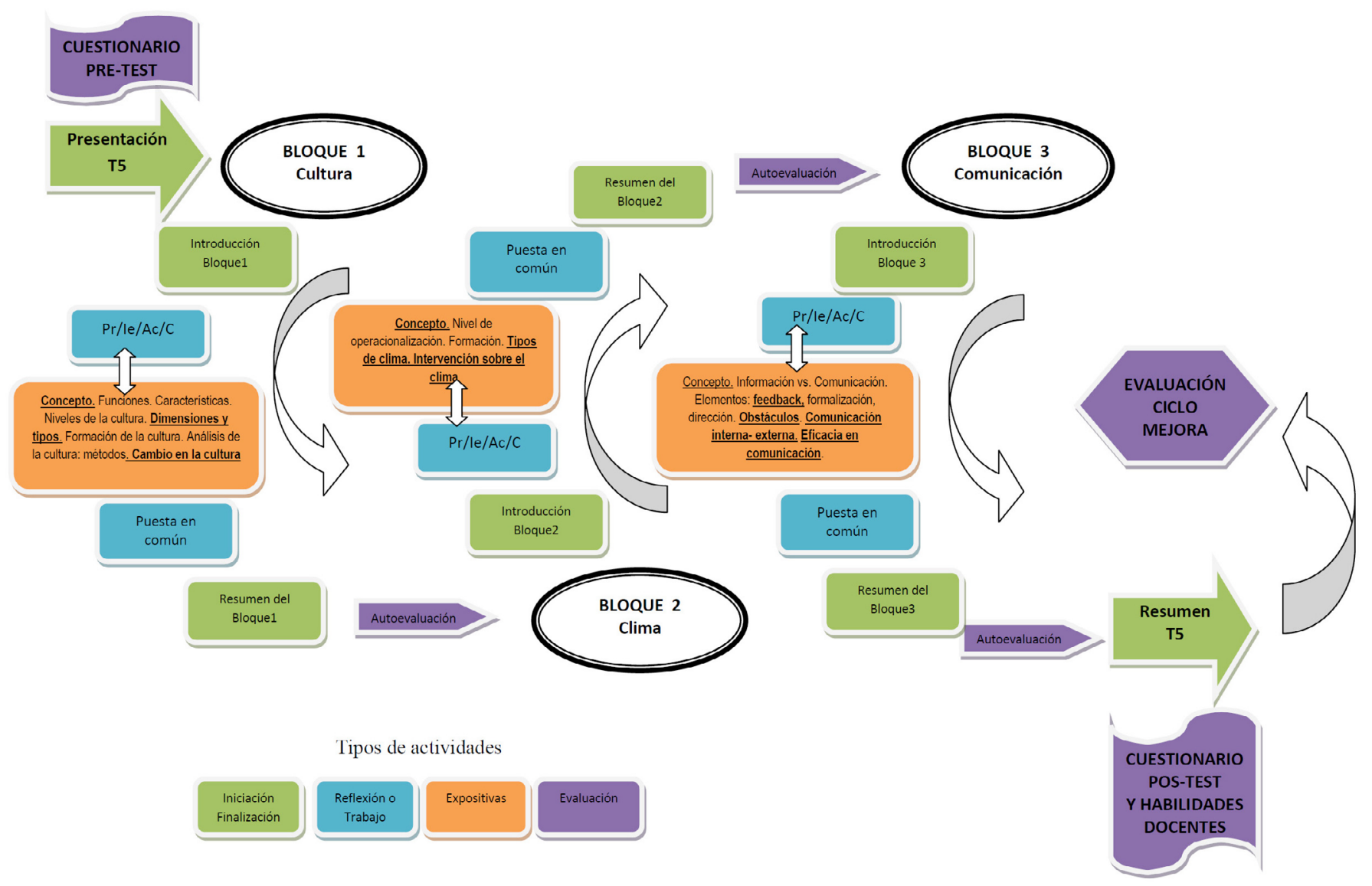

Figura 1. Mapa de contenidos y actividades

Jornadas de Formación e Innovación Docente del Profesorado | № 1 (2018)

(C) Esta obra se distribuye con la licencia Creative Commons

(c) (i) ()$\left._{\mathrm{BY}}\right)$ Reconocimiento-NoComercial-SinObraDerivada

Internacional (CC BY-NC-ND 4.0.) 
Al terminar el tercer y último bloque se realiza un resumen general del tema y se pasa de nuevo el cuestionario final (post-test) y también el cuestionario de habilidades docentes.

En la presentación del tema el objetivo es que el alumno construya un esquema general partiendo de sus conocimientos actuales sobre cultura, clima y comunicación, esquema que más adelante se irá completando con contenidos específicos. Se trata de dar una descripción general del tema para ofrecer una visión global y fácilmente comprensible de los contenidos que se van a plantear. Se apoya con una diapositiva que recoge el indice del tema; también se realiza la proyección de un vídeo introductorio seguido de un corto debate sobre su contenido.

En cada uno de los tres bloques el objetivo es que el alumno construya un esquema propio sobre la cultura, el clima y la comunicación organizacional para ser contrastado más adelante con los conocimientos teóricos bien establecidos en este campo. De esta forma el alumno percibirá la diferencia entre su conocimiento actual y el conocimiento teórico académico, con la intención de mejorar su aprendizaje. Cada bloque, a su vez, se divide en tres partes. El resumen final ofrecerá una visión global de cada bloque en forma de esquema para asentar conocimientos. Se apoya con diapositivas, explicaciones del profesor, ejemplos prácticos y planteamiento y resolución de preguntas o casos. Cada bloque finaliza con una autoevaluación de contenidos aprendidos.

En el resumen final, el objetivo es que el alumno tenga una visión global del tema y de sus contenidos. El alumno podrá comprobar si ha comprendido la globalidad del tema y plantear sus dudas. Se trata de dar una descripción general del tema para proporcionar una visión global y fácilmente comprensible de los contenidos que se han tratado en el tema. Se apoya con una diapositiva con el índice del tema. Los alumnos plantearán sus dudas.

Finalmente, como parte de la evaluación del ciclo de mejora se pasa un cuestionario final post-test de 
evaluación de los esquemas mentales de los alumnos y alumnas, y un cuestionario de evaluación de la docencia y habilidades docentes del profesor.

\section{Evaluación}

El ciclo de mejora docente contó con cuatro tipos de evaluación que se describen en la Tabla 3: autoevaluación, evaluación pre y post de los esquemas mentales, evaluación de la docencia y habilidades docentes, trabajos realizados durante las sesiones.

Tabla 3. Tipos de evaluación realizados durante el ciclo de mejora docente.

\begin{tabular}{|l|}
\multicolumn{1}{|c|}{ Tipos de evaluación } \\
\hline $\begin{array}{l}\text { Autoevaluación de los conocimientos adquiridos en clase para cada blo- } \\
\text { que del tema. Por tanto, como el tema tiene tres bloques, los alumnos rea- } \\
\text { lizan una autoevaluación de sus conocimientos en tres ocasiones, tras cada } \\
\text { bloque. }\end{array}$ \\
\hline
\end{tabular}

Evaluación pre y post de los esquemas mentales de los alumnos. Se presenta un caso (caso de la empresa Malrollo S.A.) que han de resolver en una primera ocasión basándose en sus conocimientos sobre cultura, clima y comunicación y en una segunda ocasión al final del tema basándose en los conocimientos adquiridos en clase.

Evaluación de la docencia y las habilidades docentes del profesor.

Hojas de trabajo de cada sesión que corresponden a cada bloque del tema; se puntúan de 0 a 1 puntos. Estos puntos de los trabajos de clase se acumulan y sirven para subir la nota del examen final de la asignatura, siempre que se haya aprobado.

Los criterios de evaluación utilizados para el pretest y postest (caso de la empresa Malrollo S.A.) se recogen en la Figura 2. Para cada uno de los tres conceptos principales (cultura, clima y comunicación) se realizó un análisis cualitativo y cuantitativo de los modelos mentales de los alumnos en base a la respuesta al caso planteado. Las puntuaciones de cada concepto principal varían desde 0 a 4; por lo tanto, la puntuación total oscila de un mínimo de 0 a un máximo de 12. 
0 . Desconoce el concepto, no plantea una solución oes inadecuada.
1. Conocimiento elemental usando términos generales, no

relaciona

conceptos,

plantea una

solución general.
2. Conocimiento

básico (usa al

menos 1

concepto

teórico), usando

términos teóricos

correctos, no

relaciona

conceptos,

plantea una

solución general

adecuada.
3. Conocimiento

medio (usa al

menos 20 más

conceptos

teóricos), con

términos teóricos

correctos,

relaciona los

conceptos, no

extrae

conclusiones,

plantea una

solución

adecuada y

específica

teniendo en

cuenta el

contexto.
4. Conocimiento completo (usa la mayoria de los

conceptos

teóricos),

usando términos

teóricos

correctos,

relaciona

conceptos,

extrae

conclusiones,

plantea una

solución

adecuaday

específica

teniendo en

cuenta el

contexto y la

justifica.

Figura 2. Escalera de evaluación para pretest y postest (caso de la empresa Malrollo S.A.)

\section{Aplicación del Ciclo de Mejora Docente}

\section{Sintesis del desarrollo de las sesiones}

Respecto al clima de trabajo, el ambiente de la clase fue bueno y relajado; los alumnos no hablaban entre ellos distrayéndose y mostraron siempre una actitud participativa y de interés. Por mi parte, al percibir que los alumnos mostraban interés a lo largo de la clase, me encontré mucho más cómodo. Esto me tranquilizó bastante y facilitó que les hablase de una forma más cercana y fluida.

Los alumnos se involucraron en realizar las actividades; esto se vio reflejado en los contenidos de las actividades que entregaron al final de las clases, evidenciando esta implicación. He detectado una mayor implicación de los alumnos en este tipo de clases. Al explicarles la metodología y los resultados que ellos pueden obtener de esta, lo comprenden y se nota en su participación e interés.

Al principio cuesta hacerles comprender que este sistema de docencia es mejor que el anterior, más tradicional y expositivo. Cambiar sus esquemas de lo que ha de ser una clase "teórica" es difícil y también hacerles pensar y 
relacionar conceptos, más aún si se han dado en otras sesiones anteriores.

\section{Resultados de la evaluación}

\section{Autoevaluación}

La autoevaluación consistió en calificar de 0 a 10 el conocimiento de los conceptos tratados en clase. Cero indica que actualmente no conoce este concepto y, diez indica que actualmente conoce y domina todos los conceptos al $100 \%$ y sin dudas. Esta autoevaluación tuvo dos objetivos, por un lado los alumnos al reflexionar sobre su aprendizaje de cada concepto trabajado en clase detectan las lagunas de cara a prepararse para el examen y asentar conocimientos y por otro lado informa al docente del aprendizaje de los alumnos. La media para los tres conceptos principales de este tema superó de forma ajustada el aprobado pero no logró una nota elevada. Estos resultados reflejan que los alumnos tienen conciencia de que aún les queda por asentar conocimientos tratados en clase de cara al examen y a completar su aprendizaje de la matera. Por tanto, y según las percepciones subjetivas de los alumnos sobre su conocimiento aprobarían el examen pero con un mero aprobado. Hay que tener en cuenta que esta autoevaluación es una percepción subjetiva del alumno respecto a sus conocimientos adquiridos.

\section{Comparación de los cuestionarios de pre-test y pos-test}

Los gráficos de las Figuras 3, 4 y 5 representan el porcentaje de alumnos que se encontraba en cada escalón de la escalera de valoración del cuestionario de pre-test y pos-test anteriormente explicados. Como se puede observar, en el pre-test la mayoría de los alumnos se encontraba en el escalón 1 correspondiente a un nivel elemental en los tres conceptos del tema (cultura, clima y comunicación). En cambio en el pos-test se observa una evolución 
positiva sobre todo en cultura y comunicación, pasando de un nivel elemental a un nivel básico o medio. Hay que señalar que ningún alumno llegó al nivel 4.

\section{Cultura}

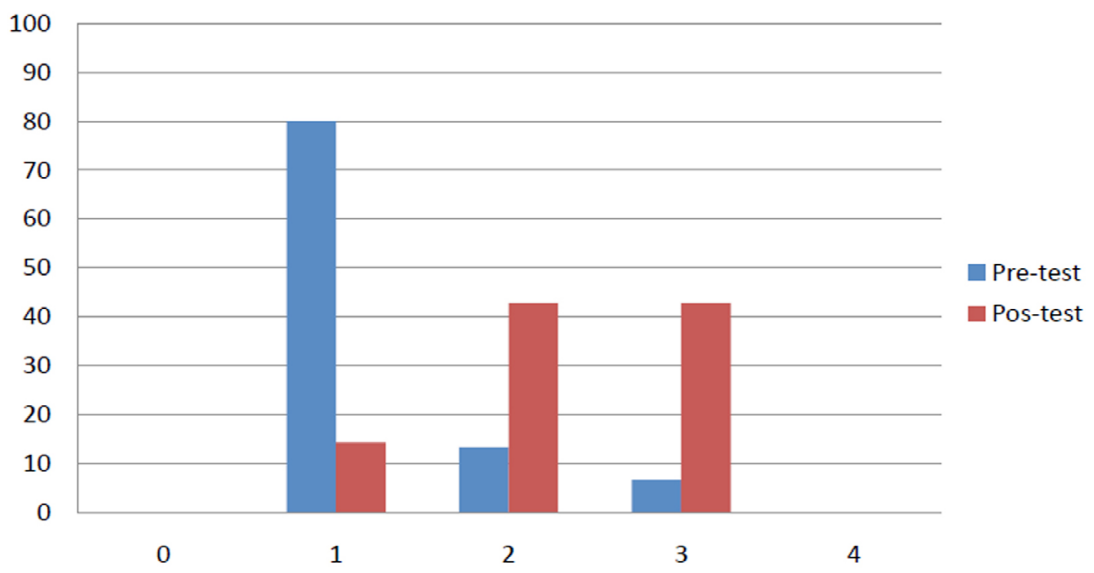

Figura 3. Puntuación en \% de pre-test y pos-test del concepto cultura.

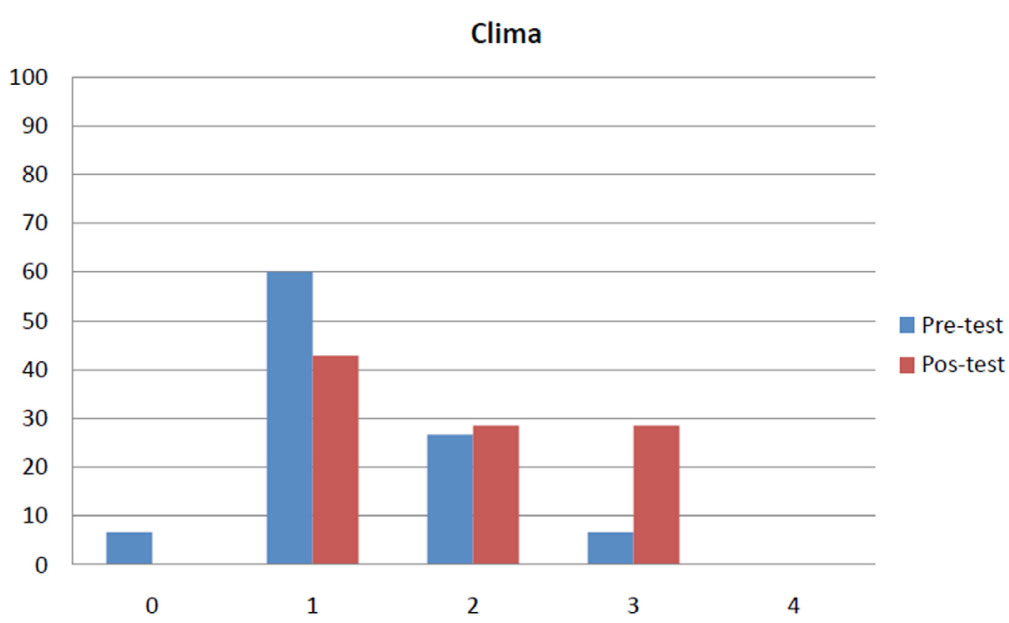

Figura 4. Puntuación en \% de pre-test y pos-test del concepto clima.

Jornadas de Formación e Innovación Docente del Profesorado | № 1 (2018)

(c) (i) (2) Esta obra se distribuye con la licencia Creative Commons Reconocimiento-NoComercial-SinObraDerivada $\quad 4.0$ Internacional (CC BY-NC-ND 4.0.) 
Comunicación

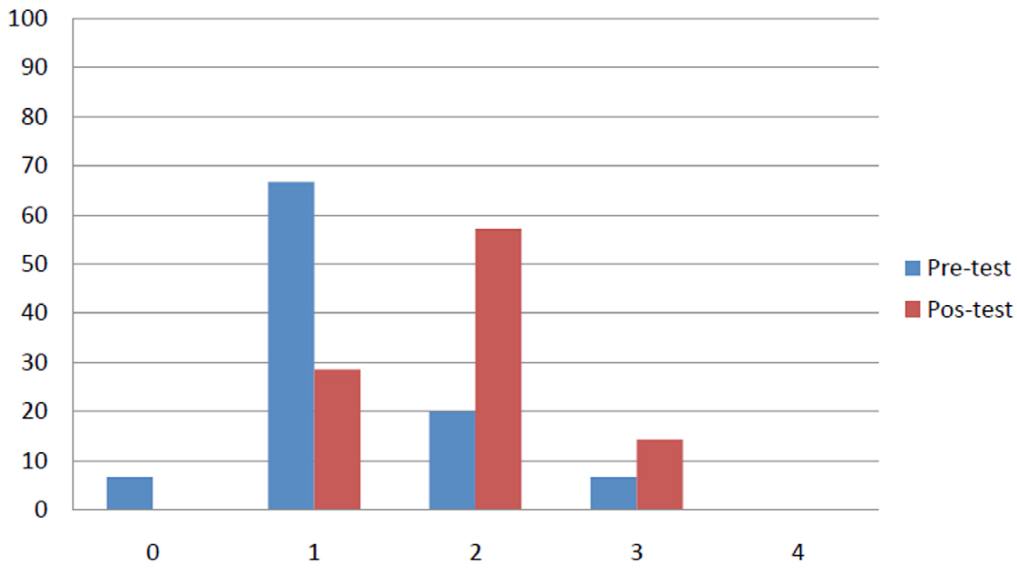

Figura 5. Puntuación en \% de pre-test y pos-test del concepto comunicación.

\section{Habilidades docentes del profesor y conocimientos de los alumnos}

El gráfico de la Figura 6 muestra dos tipos de información. Por una parte, las barras de P1 a P9 reflejan la media de las respuestas a las preguntas sobre habilidades docentes basadas en los principios propuestos por Ken Bain (2007). Las preguntas fueron las siguientes: P1: Crea un entorno adecuado para el aprendizaje; P2: Consigue captar y mantener tu atención; P3: Conecta el conocimiento teórico con la realidad; P4: Despierta tu interés por el conocimiento académico teórico; P5: Crea experiencias de aprendizaje diversas; P6: Tiene en clase una buena oratoria, estimulante y clara; P7: Usa un lenguaje que implica intelectual y emocionalmente a los alumnos/as; P8: Explica de lo general y sencillo a lo específico y complejo de forma gradual; P9: Permite la participación y el intercambio de ideas de los alumnos/as. Por otra parte, las barras de T1 a T6 reflejan la media de los conocimientos que los alumnos consideran que poseen de cada uno de los temas que componen la asignatura: Tema 1: Análisis de las aportaciones de la Psicología a la explicación del comportamiento en las organizaciones; Tema 2: Pensamiento, percepción interpersonal y cognición social; Tema 3: El significado del 
trabajo y actitudes hacia el trabajo; Tema 4: Equipos de trabajo, liderazgo y poder; Tema 5: Cultura y clima y comunicación organizacional; Tema 6: Riesgos psicosociales en el trabajo. Las respuestas a las preguntas pueden oscilar de un mínimo de 1 a un máximo de 5. Esta información se refiere a percepciones subjetivas de los alumnos sobre las preguntas que se le plantean, y los valores son medias del conjunto de los alumnos, existiendo casos con puntuaciones por encima y por debajo de estos valores medios.

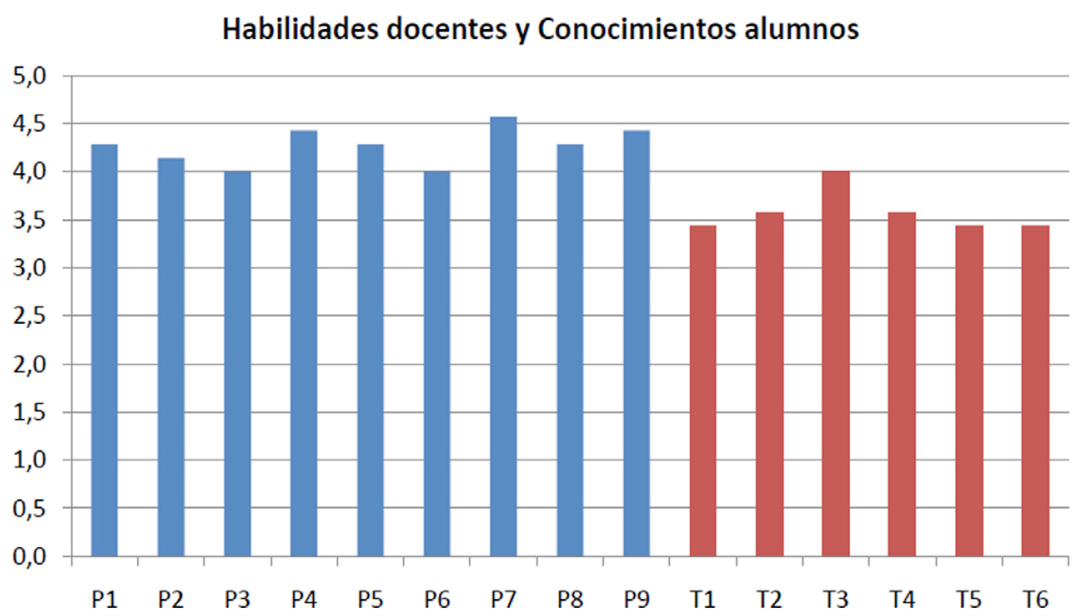

Figura 6. Medias de habilidades docentes del profesor (azul) y conocimientos de los alumnos (rojo). Escala mínimo 1, máximo 5.

En relación con las habilidades docentes, se observa que en todas las preguntas la puntuación llega como mínimo al 4 sobre 5 , indicando así unas habilidades notables. En relación con los conocimientos de los alumnos, observamos que se acercan al 3,5 sobre 5, indicando también un conocimiento notable. En principio y con la precaución de considerar que se trata de medias de medida subjetiva, teniendo en cuenta la relación entre ambas variables (habilidades docentes y conocimientos adquiridos), estoy satisfecho con la evaluación que han realizado los alumnos y eso me anima a seguir mejorando.

Jornadas de Formación e Innovación Docente del Profesorado I № 1 (2018) Esta obra se distribuye con la licencia Creative Commons 


\section{Evaluación personal del Ciclo de Mejora Docente}

Refiriéndome, por último a las cuestiones a mantener y a los cambios a introducir, tras la aplicación del ciclo de mejora, considero que mantendría, desde luego, el modelo de reelaboración de ideas de los alumnos planteado en el capítulo 2 del libro Enseñanza universitaria. Cómo mejorarla (De Alba y Porlán, 2017), ya que he podido comprobar de primera mano que el hecho de plantear problemas basados en los contenidos mejora notablemente la toma de conciencia de los alumnos sobre la materia, lo que más tarde se contrasta con el conocimiento académico establecido. Este modelo ha demostrado su efectividad en mejorar el aprendizaje de mis alumnos y ha mejorado la motivación tanto de los alumnos como la mía propia.

Por otra parte el taller conceptual, tal y como lo plantea Finkel (2008), no me parece adecuado para aplicarlo a toda la materia por cuestiones de tiempo, pero desde luego es muy recomendable incluir alguna actividad de este tipo en la asignatura, así que lo planificaré en el próximo curso.

Respecto a las habilidades docentes y dado que esta ha sido mi primera experiencia como docente universitario, he de reconocer que todavía me queda mucho por mejorar y aprender en este tema. Desde luego el conseguir captar la atención de los estudiantes y no perderla me parece fundamental y es extremadamente difícil, aunque este método de resolución de problemas me ha ayudado.

En conclusión, aún me queda mucho por mejorar respecto a las habilidades docentes propias de los buenos profesores universitarios, tal como lo plantea en su libro Ken Bain (2007). 


\section{Referencias bibliográficas}

Bain, K. (2007). Lo que hacen los mejores profesores universitarios. Valencia: Universitat de Valencia.

De Alba, N. y Porlán, R. (2017). La metodología de enseñanza. En R. Porlán (coord.), Enseñanza universitaria. Cómo mejorarla (pp. 37-54). Madrid: Morata.

Finkel, D. (2008). Dar clașe con la boca cerrada. Valencia: Universitat de Valencia.

Gil, F. y Alcover, C. M. (2003). Introducción a la Psicología de las Organizaciones. Madrid: Alianza Editorial.

Jornadas de Formación e Innovación Docente del Profesorado | № 1 (2018) Esta obra se distribuye con la licencia Creative Commons 